excellent method for designing the large synthetic arrays necessary to bridge the gap between small-molecule and macromolecule DNA-recognition motifs.

Absorption, nuclear magnetic circular dichroism, and flow linear dichroism spectra showed that while both the $\mathrm{M}$ and $\mathrm{P}$ enantiomers of $\left[\mathrm{Fe}_{2} \mathrm{~L}_{3}\right]^{4+}$ bind strongly to DNA, their binding modes and structural effects are very different; the first binding mode of the $\mathrm{M}$ enantiomer is along the major groove, and the $\mathrm{P}$ enantiomer most likely binds in the minor groove, according to the researchers. Molecular-level images obtained with atomic force microscopy are consistent with these binding modes and provided visual evidence of the induced DNA bending/coiling. The $\mathrm{M}$ enantiomer kinks and coils DNA substantially more than the P enantiomer, whose effect was significant but much less dramatic. The coiling had little effect on the local structure of the base-base interactions, a characteristic in common with molecules that serve to package DNA into chromosomes.

In current studies, Rodger and Hannon are derivitizing the M-enantiomer backbone in order to optimize the hydrogenbonding and steric effects in the major groove. Rodger and Hannon anticipate that tuning sequence selectivity into the backbone of the $\mathrm{M}$ enantiomer will enable them to target specific sequences to be coiled.

STEVEN TROHALAKI

\section{Extraordinary Magnetoresistance of InSb Allows Fabrication of a Read-Head Sensor without Magnetic Noise}

Read-head sensors are one of the main devices for data recording and storage in hard-disk drives. These sensors are commercially manufactured from magnetic materials with properties such as giant magnetoresistance (GMR) or tunneling magnetoresistance (TMR), and therefore are subject to magnetic noise. As a consequence, the use of read heads based on GMR and TMR is limited to areal densities of the order of $100 \mathrm{~Gb} / \mathrm{in}^{2}$. However, the extraordinary magnetoresistance (EMR) exhibited by nonmagnetic narrow-gap semiconductors such as InSb is free of magnetic noise and represents an alternative for a new class of read-head sensors that are compatible with large capacity storage.

A group of scientists from the NEC Research Institute in Princeton, N.J., the NEC Fundamental Research Laboratories in Japan, and the University of Oklahoma joined efforts under the guidance of NEC Institute's Stuart Solin to take advantage of the EMR properties of InSb to fabricate a mesoscopic read-head sensor, as they explained in the May 27 issue of Applied Physics Letters.
EMR appears as a consequence of a magnetic-field-induced deflection of current around a patterned metallic inhomogeneity present in or on the boundary of a semiconductor. A narrow-gap semiconductor with high-mobility carriers such as InSb shows this property in films thicker than $1 \mu \mathrm{m}$. Read-head sensors require thinner films of $\leq 100 \mathrm{~nm}$. Since carrier mobility in InSb decreases dramatically for thicknesses below $1 \mu \mathrm{m}$, this group of scientists designed a quantumwell structure to fabricate a mesoscopic sensor within the targeted thickness with a high mobility of $2.3 \mathrm{~m}^{2} / \mathrm{V}$ s at $300 \mathrm{~K}$.

The fabrication of this device was accomplished using a shadow-mask electronbeam lithography technique. A 30-nm layer of $\mathrm{Si}_{3} \mathrm{~N}_{4}$ was first deposited over the substrate, where gold strips for wiring defined an $80-\mu \mathrm{m}$-square area. In the corners of this square, a pattern of four $30 \mathrm{~nm} \times 3 \mu \mathrm{m}$ strips was delineated after a second 30-nm layer of calixarene, a new type of high-resolution resist developed by NEC, was deposited. A mesa containing the quantum well was then reactive-ion-etched using a mixture of $\mathrm{CH}_{4}+\mathrm{H}_{2}$, ensuring that the mesa walls were almost vertical. This process allowed the achievement of a structure below $100 \mathrm{~nm}$ with high alignment accuracy $( \pm 10 \mathrm{~nm})$. A suspended Ge stencil mask allowed the preparation of gold leads and a gold shunt to allow electrical contact.

Measurements at $300 \mathrm{~K}$ of the fielddependence of the observed effective resistance show a quasi-parabolic trend similar to that of macroscopic EMR plate structures. The observed maximum current sensitivity was $147 \Omega / T$, and the EMR had an optimized value of $6 \%$ at a bias field of $0.27 \mathrm{~T}$ when the applied signal field was $0.05 \mathrm{~T}$. The EMR at zero bias was $2 \%$, and is lower than the $500 \%$ obtained from macroscopic plates with higher mobility and much less leakage current bypassing the mesa. The observed resistance was $1253 \Omega$, and is controlled by the conductance of the shunt and the dopant level of the quantum well. Therefore, the researchers were able to fabricate an EMR read-head sensor using the e-beam lithography technique. The conservatively calculated areal density of the sensor, based on geometry considerations, is $116 \mathrm{~Gb} / \mathrm{in}^{2}$. At this level, estimations of the signal-tonoise ratio at $200 \mathrm{MHz}$ give $41 \mathrm{~dB}$, which is larger than estimations for a hypothetical $100 \mathrm{~Gb} /$ in. $^{2}$ TMR device at similar conditions, $34 \mathrm{~dB}$. By reducing the mesa width and voltage lead spacing by factors of 2 and 3 respectively, the research group expects to optimize the performance of such a device in the future to reach areal densities of the order of $1 \mathrm{~Tb} /$ in. $^{2}$.

SIARI S. SOSA

\section{Annular Dark-Field Scanning Transmission Electron Microscopy Captures Sb-Related Clusters in Silicon}

The electrical properties of a semiconductor can be controlled through the addition of dopant atoms to the material. However, at high dopant concentrations, the linear relationship of increased electrical activity with dopant concentration starts to break down. As silicon-based electronic devices continue to become smaller, higher charge carrier concentrations are required to maintain device performance. It therefore becomes important to understand exactly how dopant atoms become electrically inactive. To study how this happens, researchers at Bell Laboratories and Agere Systems have used atomically resolved, annular dark-field scanning transmission electron microscopy (ADFSTEM) to study antimony (Sb) dopant atoms in crystalline silicon (Si) in samples with $\sim 30 \%$ of dopants electrically inactive.

The researchers reported in the April 25 issue of Nature that they were able to distinguish between $\mathrm{Sb}$ and $\mathrm{Si}$ atoms with ADFSTEM technique because the image intensity of each species is proportional to the atomic number of the element raised to the 1.7 power $\left(Z^{1.7}\right)$. Thus, one $\mathrm{Sb}$ atom appeared approximately nine times as intense as one $\mathrm{Si}$ atom in the images, they said. The research group found that the intensity and distribution of $\mathrm{Sb}$ in the samples was well described by a binomial distribution, suggesting that $\mathrm{Sb}$ substitution in $\mathrm{Si}$ sites is random.

From the images, the research team concluded that the deactivating dopant cluster in the Si crystals contained only two Sb atoms. Paired defects outnumbered four atom clusters by 50:1. Since ADF-STEM images view structures in projection, the experiments do not allow for determining whether the primary pair defect contains a vacancy, or is of the donor pair type.

While single heavy atoms on surfaces have been visualized previously, "this is the first time individual impurity atoms have been imaged inside a crystal at atomic resolution," said David Muller of Bell Laboratories. "Because we are viewing the atoms in their undisturbed bulklike states, we can identify bulk-defect complexes,"

The key to this work lies in the TEM sample preparation, according to Muller and coworkers. Instead of using ion milling, which leaves a damaged layer a few nanometers thick, the researchers use chemical-mechanical polishing. Then an etch is used to remove any oxide. The researchers reported, "Random variations in thickness must be less than the contrast of one $\mathrm{Sb}$ atom, and the surfaces must be free from native amorphous $\mathrm{SiO}_{2}$ layers and damaged bulk Si layers amorphized during thinning."

CHRISTOPHER MATRANGA 